\title{
LA BASE Y EL ORIGEN SOCIO-CULTURAL DE LAS PSICOTERAPIAS COGNITIVAS
}

\section{THE FOUNDATION AND SOCIO-CULTURAL ORIGIN OF COGNITIVE PSYCHOTHERAPIES}

\author{
Isabel Caro Gabalda
}

Universidad de Valencia. Facultad de Psicología

Dpto. de Personalidad, Evaluación y Tratamientos Psicológicos, Valencia, España

Cómo referenciar este artículo/How to reference this article:

Caro Gabalda, I. (2017). La Base y el Origen Socio-cultural de las Psicoterapias Cognitivas. Revista de Psicoterapia, 28(107), 271-293.

\section{Resumen}

Este trabajo, de corte teórico, tiene como finalidad principal rastrear las bases y el origen socio-cultural de las psicoterapias cognitivas. Para ello, el trabajo comienza describiendo, brevemente, los elementos principales del modelo cognitivo y señalando, a continuación, las relaciones entre psicoterapia y marco occidental. Seguidamente, se procede a una comparación en mayor detalle entre las psicoterapias cognitivas y su particular origen socio-cultural. Elementos clave que seresaltan yrelacionan con las psicoterapias cognitivas son el individualismo, la búsqueda de control y autonomía, un self individual autolimitado, y la consecución del racionalismo. El desarrollo de estos apartados tiene en cuenta a las principales terapias cognitivas, de corte clásico, o de corte constructivista. Finalmente, el trabajo termina haciendo una reflexión de todo ello desde la perspectiva del sujeto cognitivo.

Palabras clave: psicoterapias cognitivas, sociedad occidental, individualismo, racionalismo, sujeto cognitivo

\begin{abstract}
This theoretical paper aims to describe the sociocultural foundations and origin of cognitive psychotherapies. Therefore, it begins with a brief conceptualization of main cognitive therapies elements, pointing out, thereafter, to the relationships between psychotherapy and a Western society. Then, a more detailed comparison is done between cognitive psychotherapies and their specific socio-cultural origin. Key issues to emphasize are, individualism, pursuing of control and autonomy, a bounded self, and rationalism. The development of those elements is related not only with the more classical cognitive psychotherapies, but also with the constructivist ones. Finally, an integrative reflection is made in relation to the cognitive subject.

Keywords: cognitive psychotherapies, Western society, individualism, rationalism, cognitive subject
\end{abstract}


La psicología siempre presupone una cosmología (Berger y Luckmann, 1966, pág. 196)

La historia de las psicoterapias cognitivas se ha escrito de diversas maneras. No obstante, la forma principal tiene que ver con señalar sus elementos y conceptos centrales, técnicas principales, y con dotar a este enfoque del necesario contexto histórico. En principio, no nos cabe duda de que ningún modelo terapéutico ha surgido, o puede surgir de la nada. Así, señalar por parte de los principales modelos cognitivos (Beck y Weishaar, 1989; Dryden y Ellis, 1988; Ellis, 1962) que deben, o asientan algunos de sus principios más básicos en autores como Kant, Descartes, Popper, Korzybski, Piaget, Kelly, etc., no nos debe extrañar y parece un primer paso lógico a la hora de presentar un enfoque.

No obstante, lo que no suele ser tan habitual es encuadrar los modelos y sus elementos y supuestos principales en el específico marco socio-cultural que los ampara. Cualquier modelo va a hacer patente o traslucir un particular "espíritu de la época". El propósito principal de este trabajo es el de explorar de qué elementos socio-culturales beben las psicoterapias cognitivas que sustentan y justifican su foco principal y su origen. La pregunta clave que nos hacemos sería: ¿cuál es el caldo de cultivo socio-cultural que facilitó plantear y certificar el modelo cognitivo? (con independencia de críticas desde dentro -cf. Mahoney, (1988) que no dejaban de ser beneficiadas, en nuestra opinión, del mismo caldo de cultivo).

Como profesionales clínicos, investigadores y académicos no podemos escapar del marco socio-cultural al que pertenecemos. Lo "cognitivo", en terapia, no se ha hecho, ni ha surgido, en un vacío. Enmarcando esto en el marco del construccionismo social (Berger y Luckmann, 1966) podemos adoptar una perspectiva mediante la cual comprendamos que el investigador que pone en marcha un estudio para comprobar la "veracidad" de la hipótesis cognitiva, por ejemplo (véase, Clark, Beck \& Alford, 1999), no puede dejar de comprobarla más que dentro de su cultura de origen, al igual que el académico o clínico que escribe sobre ella. Es decir, no tendría sentido una teoría psicológica, siguiendo el ejemplo que plantean Berger y Luckmann (1966), sobre la posesión demoniaca para interpretar los problemas de identidad de intelectuales judíos, neoyorkinos, de clase media. Como no tendría sentido emplear el psicoanálisis para interpretar los problemas de identidad en Haiti. Puestas así las cosas, parece interesante obtener cierto conocimiento sobre aquello que empleamos y el motivo ("socio-cultural") por el que lo hacemos. Funcionamos como clínicos, de la manera que lo hacemos, porque, por decirlo con pocas palabras, "no tenemos más remedio". No es una postura determinista, sino interpretativa que pretende arrojar cierta comprensión de nuestro modelo terapéutico. Para poder acceder a este fin, debemos centrar el foco de inicio. 


\section{¿Qué significa hacer psicoterapia cognitiva?}

El modelo cognitivo está bien representado en diversos enfoques y autores. Nombres como los de Beck y Ellis, Mahoney o Guidano, señalan, indudablemente, el alcance y rasgos distintivos del quehacer cognitivo y representan que el enfoque ha evolucionado con el tiempo. Denominamos, en su momento, a estos modelos como siendo, o bien de corte modernista o de corte postmoderno teniendo en cuenta las características que manifestaban cuando surgieron (Caro, 1995). Modernidad y postmodernidad son términos complejos de definir y articular (Pinillos, 1997; Seoane, 2005). A efectos de exposición, podemos asumir que los primeros acogerían a los modelos de reestructuración y los cognitivo comportamentales que podríamos considerar como primeros modelos cognitivos y a los que denominaremos modelos clásicos. Mientras que los segundos, responsables en buena medida de la evolución de las psicoterapias cognitivas, podríamos identificarlos con planteamientos de corte constructivista/construccionista.

Sin embargo, y a pesar de las notorias diferentes entre ellos, es nuestra tesis principal que sus rasgos más destacados beben del mismo marco socio-cultural y se siguen manteniendo en gran medida. Comenzaremos, pues, por señalar los elementos principales de los modelos cognitivos que intentamos englobar en este trabajo, destacando, brevemente, algunos de sus elementos y de sus diferencias principales. Este va a ser un planteamiento general que, necesariamente, no puede cubrir, específicamente, todos los enfoques denominados cognitivos.

Las psicoterapias cognitivas se preocupan de cómo conocemos y cómo damos significado a nuestras experiencias. Ya sea mediante un énfasis en la razón o en la emoción, para los terapeutas cognitivos los seres humanos conocemos de forma activa, interpretando nuestra "realidad". Por este motivo, una preocupación indudable de cualquier teórico cognitivo es la de ofrecer una conceptualización, primero, que dé cuenta de cuáles son los elementos principales que influyen en nuestra manera de conocer y de interpretar. Las respuestas ofrecidas por los psicoterapeutas cognitivos a ello son bien diversas y van a depender del marco epistemológico, ontológico, etc. en el que se articule cada enfoque cognitivo (Mahoney, 1988, 1991).

Para los modelos clásicos, la metáfora del ser humano como un científico puede ser válida. Esta metáfora se relaciona con un individuo que detecta qué le está causando problemas, fundamentalmente su marco cognitivo en forma de pensamientos y esquemas negativos, por ejemplo. Este marco cognitivo se somete, de forma racional, a validación con toda una amplia serie de técnicas. Los pensamientos, por ejemplo, se convierten en hipótesis que se someten a contrastación, buscando el soporte empírico. Es decir, el conocimiento se puede hacer más certero y mejor sustentado, representando de forma fiable y correcta la "realidad". Y así, el terapeuta guía (como un maestro que domina una materia) y el paciente le sigue (aprendiendo, motivado, de este maestro), convirtiendo la terapia en una psicoeducación. 
El científico de corte constructivista se aleja de la posibilidad de contrastación y validación. Al negar la posibilidad de encontrar base epistemológica que afirme la verdad indudable del conocimiento, éste nunca va a poder representar de forma cierta y fiable la "realidad". No existe, por tanto, un conocimiento certero, sino que la ausencia de base de este conocimiento es clave (Polkinghorne, 1992). El paciente no acude a aprender, y al terapeuta no se le ocurre jugar el papel del maestro. Finalmente, un elemento clave del constructivismo radica en la negación de la visión racionalista del sujeto, asumiendo otra de corte constructivista y narrativo. Desde esta perspectiva el individuo no es una máquina que debe procesar mejor la información, sino un contador de historias que se enfrenta a una serie de problemas dentro de una historia de vida que se ha vuelto anómala y cuyos esfuerzos se dirigen a dotar de sentido y significación a dicha historia (Gonçalves, 1994).

Las psicoterapias cognitivas presentan diversos grados de estructuración y directividad. Disponemos de terapias altamente estructuradas, como el modelo de Beck o el de Ellis, por ejemplo, lo que las hace modelos de corte teleológico, a otras de corte más teleonómico, cuyo grado de estructuración y directividad es mucho menor, por ejemplo, los modelos de Mahoney o de Guidano. Relacionado con esta cuestión tenemos el estilo del terapeuta que va a comportar una mayor o menor directividad. En este sentido, y aunque es una cuestión difícil de resumir, los modelos clásicos buscarían el control y la disminución de los síntomas que presenta el paciente, mientras que los modelos constructivistas están más inclinados a una exploración y potenciación del desarrollo del individuo, sin evitar el control y la desaparición de los síntomas (Guidano, 1997).

Un elemento clave de cualquier modelo terapéutico es el desarrollo de una serie de técnicas coherentes con su marco teórico, foco, conceptualización de casos, etc. Esta cuestión es compleja de enjuiciar, ya que la variabilidad de modelos y, sobre todo, la pericia y profesionalidad de los terapeutas cognitivos va a hacer que empleen más o menos técnicas terapéuticas. Como modelos teórico-clínicos distintivos y surgidos con una impronta diferenciadora frente a otros enfoques, las psicoterapias cognitivas explican los problemas de los seres humanos como problemas de conocimiento y se encaminan, como hemos visto, a modificarlo. Sin embargo, es notable comprobar cómo las psicoterapias cognitivas han empleado y emplean un amplio rango de técnicas. Esto supondría defender una práctica, que no un modelo, caracterizada por un cierto eclecticismo técnico (Norcross, 2005; Norcross, 1986), en donde tienen cabida técnicas provenientes del marco conductual, humanista, interpersonal, etc. Apreciamos todo ello en modelos como el de Beck (véase, Beck, Rush, Shaw \& Emery, 1979), el de Young (véase, Young, Klosko y Weishaar, 2003), o el de Mahoney (véase, Mahoney, 2005), por citar sólo unos pocos ejemplos.

Con independencia del volumen de técnicas, parece evidente asumir la imposibilidad de hacer psicoterapia cognitiva al margen de una buena relación y alianza terapéutica. El trabajo debe ser conjunto (por ejemplo, mediante el principio 
del empirismo colaborativo) y con un buen vínculo, aunque haya una mayor o menor directividad. Este vínculo garantiza la posibilidad del progreso en terapia y de que el paciente siga al terapeuta en lo que éste le propone, por ejemplo, tareas extra-sesión de diversos tipos o actividades bien diversas dentro de ella, tales como: registro de pensamientos automáticos, búsqueda de la lógica incorrecta y de pensamientos alternativos, registro de evaluaciones y debates lingüísticos, búsqueda de narraciones y construcción y deconstrucción de ellas, someter acontecimientos significativos a un foco interpretativo/narrativo, hacer y practicar exploraciones del sí mismo frente a un espejo, trabajar con el cuerpo, etc.

Para concluir esta breve descripción, todo ello supone plantear un marco y un foco terapéutico, en el que se asume que un paciente puede y debe:

1. Mirar en su interior.

2. Acceder a su marco interpretativo, comprendiendo su papel en sus problemas.

3. Trabajar con dicho marco, dándole la vuelta, modificándolo, ya sea haciendo un conocimiento más válido o más viable.

4. Establecer un vínculo de trabajo con el terapeuta.

Hecha esta breve introducción al modelo cognitivo, vamos a centrarnos en el foco principal de este trabajo. Comenzaremos relacionando la psicoterapia con la sociedad y la cultura occidental. Una vez hecho esto, entraremos a conectar más específicamente las psicoterapias cognitivas con los valores propios de la sociedad occidental relacionados con su origen.

\section{Psicoterapia, sociedad y cultura}

Sociedad y cultura son términos complejos y de compleja definición. Para nuestros propósitos convendría señalar que básicamente la sociedad sería un proceso, mientras que la cultura sería el contenido producido y utilizado en el proceso (Rychlak, 2003). El concepto de sociedad se puede construir como siendo "la unidad más grande de una población territorialmente vinculada, multigeneracional, conseguida, sobre todo gracias a la reproducción sexual, y que está organizada alrededor de una cultura común y de un sistema social común" (Rohner, 1984, p. 131).

Por su parte, Pinillos (1997) define la cultura como:

"El estilo y los medios de vida propios de toda sociedad humana. Es, por lo tanto, el sistema de valores y metas vigentes en toda comunidad humana a cuyo servicio se hallan las técnicas materiales de alimentación y defensa, y para cuya consecuencia hay que atenerse a usos y normas comunes. La cultura es, pues, el contenido del comportamiento humano en cuanto tal, esto es, algo puesto por el hombre y no meramente dado por la Naturaleza de forma instintiva; es una creación humana que regula la propia vida del hombre y la potencia" (p. 42).

Así, la cultura incluye los logros y productos de los seres humanos, los significados aprendidos y transmitidos socialmente $\mathrm{y}$, finalmente, lo material, 
espiritual, comportamental y valorativo del ser humano. Como plantea Rychlak (2003, pág. 45), la cultura acoge todo aquello que es importante para la identidad del colectivo social o de la sociedad. Así, incluiría elementos tan importantes y aparentemente dispares como, los sistemas de lenguaje, las organizaciones políticas, el conocimiento científico, los valores y sistemas de creencias, los mitos, las costumbres, la forma de vestir, el almacenamiento de logros, etc.

Estas definiciones nos ayudan a plantear un marco interpretativo que puede influir en la comprensión de nuestro trabajo. Parece evidente plantear que la psicoterapia, como disciplina científica y como práctica profesional, no surge, ni se practica, en un vacío. Detrás de la psicoterapia existe un marco de creencias, valores, premisas dadas por supuestas, etc. O como dijo Ehrenwald (1969), "la psicoterapia .... es arrastrada por sus corrientes visibles o subterráneas" (pág., 14, de la edición española).

Los psicoterapeutas no acostumbramos a plantearnos las bases sociales de nuestra disciplina y éstas tenemos que tenerlas en cuenta para entender, no sólo el desarrollo de la psicoterapia, sino también para, por ejemplo, comprender el "surgimiento" de los trastornos mentales (Gónzalez Pardo \& Pérez Álvarez, 2007). Sin embargo, en determinados focos se ha asumido, tradicionalmente, que las ideas científicas sociales están conformadas por científicos sociales que interactúan con las ideas de otros científicos sociales. Estos individuos, en interacción, forman parte de una comunidad más amplia -la sociedad en general-, por lo que sus ideas y evolución reflejan e influencian la estructura social (Buss, 1975). No es nueva, por tanto, la idea de que la psicología y la sociedad se moldean la una a la otra, por lo que creencias culturales y morales se van a ver reflejadas en la psicología tanto a niveles teóricos, como prácticos (véase un resumen en Prilleltensky, 1989). De forma más específica, cualquier sistema de psicoterapia va a reflejar lo que es correcto, e incorrecto, estableciendo, por tanto, una agenda relacionada con aquello que quiere modificar, disminuir y permitir (Owen, 1993). Esta agenda es, a su vez, un proceso y un producto socio-cultural.

En este sentido, y siguiendo a Robinson (1997), la terapia supone a la vez, una teoría que tiene, además, una influencia cívica. La teoría se refiere a los tipos de vida que merece la pena vivirse, la identidad a la que debemos de aspirar, y a los modos y maneras por los que una persona puede influir en los otros. Es decir, que la psicoterapia va más allá de ser una teoría que está asentada en una escuela o sistema teórico concreto. La psicoterapia es una teoría sobre las personas, lo que supone entender lo que es correcto para ellas, en su vida y en sus aspiraciones. Igualmente, la psicoterapia tiene consecuencias, ya que anticipa los efectos de los clientes sobre otras personas, al disponer (seleccionando, organizando y orientando), de aquellas iniciativas por las que los pacientes influyen en su mundo.

Estas "teorías" y el rango de su influencia no son consecuencia de un azar, sino de un proceso complejo en el que inevitablemente nos enmarcamos. Desde un punto de vista construccionista social, tanto las categorías diagnósticas, como la psicote- 
rapia son construcciones sociales que son definidas socialmente y que desde este punto de vista serán irrelevantes al margen de otras culturas (Eberle, 1993; James $\&$ Prilleltensky, 2002). Cada sociedad nos ofrece y define un modelo y una realidad psicológica (Berger, 1965). Para Cushman (1993), la psicoterapia es un producto cultural, y en este sentido, refleja y reproduce su contexto cultural, o el contexto cultural en el que surge.

\section{Psicoterapia y sistema occidental}

Diversos autores han descrito a este contexto, como un contexto que articula $\mathrm{y}$ hace suyos los principios y valores de la sociedad occidental y ha contribuido a su desarrollo (Cushman, 1992; Hansen, 2014; James \& Foerster, 2006; Toukmanian \& Brouwers, 1998). Es un comentario común de todos aquellos autores que reflexionan sobre el marco socio-cultural de la psicoterapia que ésta y acogiendo, también, los principales sistemas terapéuticos, no deja de ser un reflejo del mundo occidental, europeo y americano (Messer y Woolfolk, 1998; Wampold, 2001; Watts, 1961), tal y como luego desarrollaremos en relación a las psicoterapias cognitivas. Y ello se ve no sólo en el sustrato teórico, sino en cada uno de sus integrantes.

En su momento, Calestro (1972, pág. 97; citado en Dow, 1986, pág. 60) describió el mito del terapeuta occidental de la siguiente manera:

"Las creencias del terapeuta en relación a su eficacia como agente de cura derivan de su formación y adopción de una escuela concreta de psicoterapia. Se le enseña a creer que el malestar emocional o las anomalías conductuales se desarrollan en función de ciertos principios sistemáticos y científicos. Se le enseña, también, que principios semejantes se pueden emplear para corregir las anormalidades psicológicas. Estas creencias, consistentes con su mundo de supuestos, organizan toda la sustancia de su mito personal".

Este mito es compartido por el paciente, ya que el psicoterapeuta occidental le pide al paciente que dramatice y proyecte su propio mundo mítico (Dow, 1986). Como planteó Eberle (1993) las teorías psicológicas tienen efectos socializantes y modulan identidades, más que otro tipo de teorías. Las terapias sirven a ideologías específicas, produciendo realidades que son la base de su propia verificación.

Para seguir desarrollando esta cuestión debemos señalar que el modelo cognitivo hace suyos los siguientes principios: individualismo y trabajo con "lo interior"; racionalismo; responsabilidad y autonomía y logro del control. Esto tiene una importancia clave para la psicoterapia y nuestra comprensión de la psicoterapia cognitiva, por dos motivos: situar el "ideario moral" a su base, y no olvidar que para que la psicoterapia funcione (Frank, 1961) paciente y terapeuta comparten un mito y ritual. Esto es un camino de doble dirección. Vivimos en una sociedad imbuida, a su vez, de los modos y maneras de la psicología y la psicoterapia (Throop, 2009). 
Desde la psicoterapia cognitiva a la sociedad

Hemos planteado con anterioridad y siguiendo a Prilleltensky (1989) que la psicoterapia no sólo reproduce el estatus-quo, sino que al ser un producto de su época puede influir en creencias, valores, etc. de su momento socio-cultural.

Desde un punto de vista positivo, Cushman (1993) señala que la psicoterapia, aún enraizada en la tradición occidental, puede suponer una alternativa al estatusquo. Para Cushman (op. cit.) aunque la psicoterapia se practica dentro de un marco moral individualista y consumista, a menudo suaviza dicho marco. Alega Cushman para ello diversas prácticas como respetar, escuchar y comprender al paciente, en un marco de confidencialidad, ajeno a cualquier tipo de explotación. La psicoterapia ofrece una actitud de honestidad, confianza y esperanza.

Es fácil dar una lectura desde el marco cognitivo a esta cuestión. Consideremos, por ejemplo, como hace Cushman (op. cit.) que la psicoterapia:

“... ofrece valores alternativos como el respeto por las experiencias personales y las ideas del paciente, y una creencia en el habla directa y en la importancia de comprender y empatizar con el otro valorando el autoexamen, la introspección y el logro parcial del auto-conocimiento y del insight psicológico" (Cushman, op. cit. pág. 106).

Como sistema psicoterapéutico, estos elementos se hacen evidentes en las psicoterapias cognitivas. Se asume, fácilmente, que los pacientes tienen acceso al mundo interior, contribuyendo, pues, la psicoterapia cognitiva a construir un sujeto que realiza una acción primordial, el acceso, mediante la introspección, a su mundo cognitivo (Caro Gabalda, 2015). El sujeto cognitivo puede y debe implicarse en dichas acciones (tal y como reflexionaremos al final de este trabajo).

Desde otra perspectiva se pronuncian Dobson y Dobson (2009). Estos autores reflexionan sobre la contribución y la vinculación de la terapia cognitiva con la sociedad actual. ¿Qué le ofrece la psicoterapia cognitiva a la sociedad actual que encaja a la perfección con ella?

En primer lugar, las psicoterapias cognitivas revalidan el individualismo. La argumentación básica es que si el individuo tiene problemas, en lugar de buscar el apoyo social para resolverlos, los individuos con problemas buscan terapia para aprender esas habilidades o características de las que carecen. El individuo asume que el problema está en él y busca medios para resolverlos por el camino. Dobson y Dobson (op. cit.) asumen que los defensores de las psicoterapias cognitivas valoran, de forma realista, esta búsqueda del control, del logro, del establecimiento de metas, etc. Por ello, parece que la psicoterapia cognitiva es un buen enfoque coherente con el tipo de sociedad en el que se ha desarrollado.

En segundo lugar, vivimos en un mundo en el que la información o el exceso de ella nos asaltan por todas partes. Se nos indica qué comer, cómo comer, cómo tomarnos la vida, cómo enfrentarnos a cuestiones médicas y psicológicas que suponen tomar decisiones sobre lo que debemos hacer para llevar una vida mejor, basadas en investigaciones, encuestas, materiales de divulgación, etc. La psicote- 
rapia, en cierto sentido, se ha desmitificado puesto que cualquiera tiene acceso a información sobre ella. El lego se hace cargo (Ibáñez, 1993). Los pacientes, como usuarios informados, leen y conocen lo que existe en el campo de la psicoterapia.

No sólo tenemos mayor información, sino que parece cada vez más aceptable buscar tratamiento para los problemas de salud mental. A esto ha ayudado que determinadas figuras públicas reconocieran sus problemas de salud mental. Al consumidor, cada vez más preocupado por lo que consume, se le hace llegar el mensaje de que necesita adquirir un "buen producto". Los consumidores de servicios de salud mental son un lobby que se protege a ellos mismos y a sus familias. Por ello no es de extrañar que el consumidor se incline hacia aquellos tratamientos que han sido empíricamente validados, dada la preminencia y divulgación que se les ha dado. Además, no es de extrañar que el consumidor de estos servicios busque y apoye aquellos tratamientos que adoptan un punto de vista igualitario y de colaboración activa con los pacientes. Los consumidores valoran la transparencia en terapia, lo que supone que las metas, su fundamento y los métodos a seguir estén claramente descritos. Estas cuestiones están a la base y son típicas de las terapias cognitivas, según Dobson y Dobson (2009).

En tercer lugar, hay una cuestión importante y que refleja la intersección entre la psicoterapia cognitiva y el marco socio-cultural. Nos referimos a la cuestión económica. Relacionado, además, con todo lo anterior, los consumidores y las agencias de salud dan una gran importancia al coste de las terapias y a la relación entre coste, tiempo empleado en ellas, y resultados. Dado los costes cada vez más elevados de ofrecer servicios a una población cada vez más anciana, que vive más tiempo, y el escaso número de profesionales, las agencias de salud se preocupan de ofrecer servicios que al menos den respuesta a su coste.

Un ejemplo de ello es lo que se conoce en los EEUU, como el managed care, o el pago, por determinadas corporaciones y organizaciones, de los servicios de salud. Reflexionando sobre esta cuestión Cushman y Gilford (2000) señalan como, en nuestra sociedad occidental, la corriente principal de la Psicología y los valores del mercado, reflejan creencias culturales muy enraizadas, tales como, eficiencia, pragmatismo y razonamiento objetivo. A la base algo muy importante para la Psicología: lograr una práctica efectiva mediante procedimientos científicos indiscutibles. Los problemas psicológicos, por tanto, se enmarcan en definiciones y soluciones positivistas, en coordinación con fórmulas económicas.

Creemos que se le debe dar al paciente lo que necesita (véase, por ejemplo, Mahoney, 1985) aunque con ello estamos afirmando algo que va contra el espíritu de la época. El siguiente párrafo nos da una idea muy concreta de por dónde "sopla el viento".

"Mientras amanece el siglo XXI, la psicoterapia está en una sociedad que avanza rápidamente y en la que los valores del mercado son cada vez más dominantes; estos elementos influyen en la psicología para ir hacia prácticas de terapia breve cada vez más rápidas y más eficientes, hacia categorías más concretas 
y específicas del DSM, y hacia resultados terapéuticos más cuantificables, todo para conseguir prácticas terapéuticas mucho mejor tecnologizadas e industrializadas. Estas tendencias se pueden apreciar a través de la sociedad americana; por ejemplo, George Ritzer (1993) se ha referido a ellas como la "Macdonalización" de América "(Cushman, 2002, pp. 103-104).

Si en comida, habría un gran consenso de que lo rápido y barato no es necesariamente lo mejor, ¿por qué lo asumimos en psicoterapia? Siendo esto importante, lo dejaremos para futuras reflexiones

Igualmente, los factores económicos, son también muy importantes para entender qué se investiga y qué no. En el ámbito anglosajón (y en el nuestro), donde cada vez son menores los presupuestos públicos para investigación, y los que se ponen sobre la mesa provienen de grupos concretos de presión, fundaciones y agencias privadas de investigación, el dinero va a llegar a aquellas terapias basadas en la evidencia, y que obtienen resultados a corto plazo, como las terapias cognitivas. Finalmente, la demanda social, también basada en cuestiones económicas, busca el consejo accesible y útil que sea rápido, basado en el sentido común y práctico. La psicoterapia cognitiva, ya que no es muy costosa, se puede desarrollar en un plazo relativamente breve de tiempo, ofrece resultados cuantificables y positivos y tiene unas tasas de recaídas no muy elevadas. Por tanto, las psicoterapias cognitivas (al menos las más clásicas) serían muy deseables desde esta "moral económica", contribuyendo a revalidarla.

\section{Individualismo y psicoterapia cognitiva}

El bloque básico de la psicología occidental ha seguido la tradición JudeoCristiana del individualismo que supone afirmar el foco en uno mismo y en la unicidad, autonomía, libertad y logro intrínseco del individuo y la posibilidad de lograr el control (Dobson y Dobson, 2009; Kazarian y Evans, 1998). Nuestra herencia occidental, en la revisión que hace Throop (2009) del tema, supone un individualismo y un sí mismo confinado o metido en sus propios límites (bounded self) como luego veremos.

Según Bellah et al. (1985, pág. 394 de la edición española) existen diversos significados de la palabra "individualismo". Para nuestros propósitos un individualismo expresivo sería apropiado, ya que sostiene que cada persona posee un núcleo único de sentimiento e intuición que se debe desarrollar y expresar para que se logre la individualidad. Para Bellah et al. (op. cit.) este individualismo se relacionó con el romanticismo de la cultura europea y norteamericana de los siglos XVIII y XIX y muestra afinidades, en siglos posteriores, con la cultura de la psicoterapia.

Las sociedades individualistas promocionan valores claramente terapéuticos como la auto-determinación y el desarrollo personal, la autonomía, el logro y el avance personal (James y Prilleltensky, 2002). Valores estos que son retroalimentados, a su vez, por las psicoterapias. Buena parte de las psicoterapias cognitivas ha surgido en una sociedad norteamericana que prima notablemente el 
foco de este epígrafe. "La individualidad no sólo se sanciona-es el estilo americano, el camino para convertirse en un hombre y conquistar el mundo" (Spence, 1990, pág. 341). Tradicionalmente se ha distinguido entre sociedades individualistas y colectivistas (Hofstede, 1980; Triandis, 1995), y aunque esta diferenciación se puede seguir manteniendo en algunos contextos, tenemos análisis de que esta tendencia al individualismo se está convirtiendo en otra forma de ser, en sociedades no occidentales o en grupos pertenecientes a otros grupos sociales, razas, etnias, etc. dentro de la sociedad occidental (Ogihara y Uchida, 2014; Throop, 2009).

Nuestra sociedad potencia y premia el logro individual (Markus y Kitayama, 2010). La consecuencia, es una sociedad que tiende a potenciar la consecución de la auto-estima, la auto-eficacia, el optimismo (la terapia cognitiva es optimista, en principio; Messer y Woolfolk, 1998), y la motivación intrínseca.

El modelo cognitivo supone lo que se ha llegado a considerar un enfoque tradicional, ya que su finalidad principal es el ajuste personal. Desde esta perspectiva, los valores que se preservan son los del individualismo y la libertad, estando el énfasis en ayudar al individuo y no a la comunidad (Prilleltensky, 1990, 1997). Aunque no es motivo específico de este artículo, habría que señalar que esta cuestión ha sido objeto de crítica. En este sentido, los valores típicos del individualismo son contrarios a situar el cambio en el cambio social. Los terapeutas cognitivos, con su insistencia y foco en los procesos de pensamiento, ya sean pensamientos disfuncionales o creencias irracionales, o estructuras que nos facilitan el significado, como la organización de significado personal, están especialmente inclinados a alejarse del cambio social, fomentado el individual (Prilleltensky, 1989, 1990).

Según Sampson (1981), en su influyente trabajo "Cognitive psychology as ideology", partiendo de tradiciones filosóficas como las de Kant o las de Descartes, la perspectiva cognitiva hace una doble reducción. Por un lado, tenemos la reducción subjetivista y por otro la individualista.

En la primera, la subjetivista, se da primacía a las estructuras y procesos del sujeto que conoce. Ejemplos destacados pueden ser los conceptos de esquema, de idea irracional, pensamiento distorsionado y sesgos cognitivos, tan típicos de los modelos cognitivos clásicos; o el núcleo metafísico central de los construccionistas. Por otro lado, la reducción individualista, supone centrarse en el pensamiento y el razonamiento del individuo que conoce (el famoso dictum de Descartes, "pienso, luego existo"). En el modelo cognitivo terapéutico esta reducción se aprecia en los intentos de conseguir que el individuo logre el cambio mediante el cambio en su forma de procesar, construir, dar significado, etc. a la realidad, o cambios en la dinámica Yo-Mí. ${ }^{1}$

El marco cognitivo supondría, por tanto, un egocentrismo teorético (Hogan, citado en Sampson, 1977, pág., 769) típico de la psicología americana imperante en el momento histórico en el que surgieron las terapias cognitivas, y que refleja un individualismo auto-contenido (Sampson, 1977). Traspasado este concepto plan- 
teado por Sampson al campo terapéutico cognitivo, se hace evidente que la demanda del terapeuta hacia el paciente es la de encauzarlo para que, por él mismo, resuelva, gracias a su aprendizaje en terapia o a su trabajo con el terapeuta, los problemas que está experimentando.

Creemos que nada hay en el ideario cognitivo que lo aleje del ideario individualista. El foco de análisis y de tratamiento, la forma de abordarlo, toda la conceptualización de casos y el empleo de técnicas terapéuticas suponen este individuo y enfoque individualista, al que se le provee con la guía necesaria para lograr autonomía, desarrollo y avance personal (por ejemplo, Ellis, 1973). Como diría Sampson (1981, pág. 735) lo cognitivo relega la potencia individual al mundo interior de la gimnasia mental.

El enfoque cognitivo, incluyendo el constructivista, supone un trabajo terapéutico con un foco en el cambio en el individuo, y por medios totalmente individuales. Es cierto, que el modelo constructivista alega presupuestos del construccionismo social (Berger \& Luckmann, 1966), pero, en modo alguno los desarrollan. Podemos asumir que para todo aquel que lea sus trabajos esto se hace muy evidente. Por ejemplo, la técnica de la moviola de Guidano (1991a) representa un sujeto que, convirtiéndose en escenógrafo de su propia vida, reflexiona, analiza y comprende lo que le pasa, haciendo obvia la dinámica Yo-Mí. Indudablemente, existe una gran diferencia entre esta técnica constructivista y otra de tipo racionalista, como el análisis de la lógica incorrecta, pero en ambos casos, se trata de un individuo que trabaja con él mismo para producir un cambio en él mismo.

\section{El sí mismo a la base de las psicoterapias cognitivas}

Anteriormente, hemos relacionado el individualismo con un sí mismo metido en sus propios límites. Según Markus y Kitayama (1991) la perspectiva occidental, norteamericana y europea nos plantea un sí mismo como una entidad que es autónoma, distinta, auto-suficiente y que posee disposiciones únicas. Siguiendo a Sampson (1977) la salud mental se considera como un ideal individualista y autosuficiente. Si se tienen las características adecuadas se logrará aquello que podemos considerar una meta terapéutica última: la auto-actualización, la autonomía y el dominio. Todo ello es propio de un sí mismo referencial (Landrine, 1992) que valora, ante todo, el individualismo y la autonomía y que se convierte en el centro del darse cuenta y de la auto-reflexión.

No obstante, según Erwin (1997) no todos los modelos y autores asumirían como meta principal de la psicoterapia el logro de la autonomía. Por ejemplo, los autores cognitivos y cognitivo-conductuales estarían más preocupados por la desaparición de los síntomas en cuanto a lo que constituye el problema principal. Sin embargo, superando cuestiones concretas, buena parte de los terapeutas asumiríamos que un paciente autónomo, independiente, que sabe lo que necesita y dónde falla, y cómo emplear los medios para ponerle remedio, es un fin más que deseable, ya sea a corto o a largo plazo. Esta autonomía se logra dentro de las 
posibilidades que nos ofrece la reflexión racional, el autoconocimiento mediante la introspección (Beck, 1976), o mediante la auto-observación (Guidano, 1991a), el logro del auto-control y la posibilidad de disminuir o eliminar lo que es defectuoso. Subyace a esto la idea de un "sí mismo agente" que nos lleva a considerar que nuestros pensamientos controlan nuestros estados de ánimo y nuestras acciones (Lam, 2008) y sobre ello debemos intervenir.

Este trabajo, en el marco de las psicoterapias cognitivas, se logra en el trabajo privado que, indudablemente, la interacción paciente-terapeuta favorece. El individuo usa sus capacidades y cambia, en el mejor de los casos. Para ello necesita al terapeuta, es cierto, pero el terapeuta busca que el paciente potencie lo que tiene como individuo, por él mismo.

Markus y Kitayama (1991) partiendo de trabajos de autores como Geertz, Sampson o Shweder y LeVine, plantearon dos tipos de sí mismos: independiente e interdependiente. Centrémonos en el independiente:

"Para lograr el objetivo cultural de la independencia se necesita construirse uno mismo como un individuo cuya conducta se organiza y se le da sentido sobre todo en referencia a su propio repertorio interno de pensamientos, sentimientos y acción, más que en relación a los pensamientos, sentimientos y acciones de los otros. Según esta construcción del sí mismo, y tomando prestada la frase de Geertz (1975) tan citada, la persona se ve como "un universo motivacional y cognitivo con límites, único, más o menos integrado, un centro dinámico de conciencia, emoción, juicio y acción organizada en un conjunto distintivo y establecido, de forma contrastante, tanto contra los otros como conjunto, como contra el contexto social y natural"(pág. 48)" (pág. 226).

Las psicoterapias cognitivas clásicas hacen evidente este sí mismo independiente, ya que prima lo privado (habilidades, pensamientos y sentimientos), lo único, la promoción de metas propias, de expresar el sí mismo, etc. Pero, ¿sólo los modelos cognitivos clásicos asumirían este sí mismo independiente?

En su evolución hacia planteamientos constructivistas las psicoterapias cognitivas integran otras tradiciones y conceptos que parecen alejarlas de este sí mismo individualista. La auto-definición que plantean Markus y Kitayama (op. cit.) de aquellas construcciones interdependientes del sí mismo establece la importancia de las relaciones con los otros en contextos específicos. Encontramos un paralelismo, aunque salvando las distancias, entre esta cuestión y las teorías del apego retomadas por Guidado y Liotti (1988) para describir buena parte de la psicopatología humana, o la comprensión de uno mismo mediante el ejercicio de la heterorreferencialidad (Guidado, 1991a). No obstante, otros elementos de este sí mismo interdependiente que señalaron Markus y Kitayama (1991) no se hacen patentes en el marco constructivista cognitivo. Por ejemplo, que sea más importante el mundo de lo externo, de lo público que de lo interno, de lo privado; tener como tarea promocionar las metas de otros, ocupar el lugar adecuado y comprometerse en acciones adecuadas; restringir el sí mismo y vivir en armonía con el contexto 
social, etc.

Por tanto, y manteniendo las diferencias de conceptualización de los distintos modelos cognitivos en su evolución, podemos utilizar planteamientos de algunos teóricos del sí mismo cuando describen el sí mismo típico de la cultura occidental, a pesar de la complejidad del campo (véase, Seoane, 2005). Estas teorías nos posibilitan describir el tratamiento que se le da al paciente en psicoterapia cognitiva (y en la inmensa mayoría de psicoterapias). O dicho de otro modo, describen al paciente que se necesita en terapia cognitiva. Describen el rango de lo que es importante y que va a ser el foco de trabajo. Para ello se necesita un sí mismo que sea "un universo cognitivo y emocional, el centro del darse cuenta, la emoción, el juicio y la acción" (Landrine, 1992, pág. 403). Correspondiendo a un sí mismo referencial, que señala Landrine (op. cit.) éste es necesario para el trabajo cognitivo, de cualquier tipo. Y para ello, vamos a emplear, fundamentalmente, la razón, aunque no solo, que propicia el control, amparada en todo un deseo de conseguir y de desarrollar un extenso bagaje tecnológico.

\section{Racionalismo, control y psicoterapias cognitivas}

Por un lado, un elemento clave para comprender el marco socio-cultural de origen de las psicoterapias cognitivas, es el racionalismo subyacente a buena parte de ellas. El racionalismo típico del modelo cognitivo deviene de las tradiciones del pragmatismo y el funcionalismo, (siendo el funcionalismo la contrapartida psicológica del pragmatismo filosófico, Bem \& Keijzer, 1996). Por otro lado, en psicoterapia cognitiva se hace evidente la ética protestante (Sampson, 1981). En este sentido, la terapia cognitiva (y cognitivo-conductual) supone un ser humano que es objetivo y solucionador de problemas (Messer \& Woolfolk, 1998). Podemos inferir que de ahí se deriva el énfasis en las técnicas, así como la importancia dada a la demostración de su eficacia (Messer \& Warren, 1990).

Uno de los elementos principales, por tanto, que se infieren del marco cognitivo, tiene que ver con este "racionalismo". El racionalismo "es el sistema epistemológico que afirma que todo el conocimiento es el resultado de un análisis racional de la evidencia del sentido y que esta misma evidencia no puede recogerse salvo mediante un principio directivo racional" (Robinson, 1981, pág. 206).

La visión del sujeto auto-consciente y racional ha sido criticada (Seoane, 2005). Lo racional, por ejemplo, no siempre impera a la hora de tomar una decisión. En la actualidad, incluso, se llega a analizar el ser humano como un individuo egoísta que sólo se implica en aquello que le trae algún beneficio de tipo personal y que en función de ello toma decisiones (Shirrmacher, 2014). Sin embargo, tenemos que hablar de lo racional para explicar el origen de las psicoterapias cognitivas.

Para Bem y Keijzer (1996) "la revolución cognitiva hizo una nueva ciencia a partir de la vieja idea racionalista de la mente" (pág., 449). Desde este punto de vista, que no es ajeno, ni está al margen del planteamiento individualista, la cognición es 
razonamiento, hacer inferencias, y construir un sistema cognitivo caracterizado por un sujeto que sería una "máquina inferencial" (Cummins en Bem y Keijzer, p. 451). Por ejemplo, el sine qua non de la terapia cognitiva es el diálogo socrático y la comprobación empírica de hipótesis (Hollon \& DiGiuseppe, 2010).

Este racionalismo es muy evidente en las terapias cognitivas clásicas, pero no así, en las de corte constructivista. De manera, que, en su evolución, las terapias cognitivas se alejan de una perspectiva racionalista, asumiendo principios de las teorías motrices de la mente, la epistemología evolutiva, etc. (Mahoney, 1988, 1991; Neimeyer, 1993). En estos planteamientos lo importante es la capacidad autoorganizadora del cerebro humano, viéndose a la cognición como "la capacidad del cuerpo-mente para tomar parte en un acoplamiento estructural con el ambiente" (Bem \& Keijzer, 1996, pág., 456).

Aunque el modelo cognitivo en su vertiente terapéutica no se sustenta directamente en la psicología cognitiva (Brewin, 1989; Dowd, 2002; Ibáñez, 1990; Ingram y Kendall, 1986), sí que es cierto que "el espíritu cognitivo" que imperó en la Psicología a mediados del siglo pasado (véase Mahoney, 1991) pudo contribuir con un "caldo de cultivo" que facilitó poder hablar de "eventos privados" que guiaban nuestra vida psíquica y nuestra conducta. La cuestión clave que nos permite hacer estas inferencias tiene que ver con el uso de la etiqueta "cognitiva" en la denominación de la terapia.

Siguiendo, pues, las derivaciones del término "cognitivo" nos encontramos con la importancia para el individuo de recibir información, procesarla y almacenarla lo que conlleva el consecuente control de las capacidades humanas (Smith, 1997). Aplicado a estas terapias de tipo "cognitivo", se supone que es necesario emplear nuestros procesos de pensamiento de la manera más eficiente ("racional") posible. Siguiendo principios cartesianos, sólo la mente pensante, la auto-reflexión, produce seguridad en el individuo (Seoane, 2005, pág. 43). Asumir las "teorías de la mente" nos convierte en tecnólogos eficientes que destacan el dominio técnico y el control activo sobre la naturaleza (Sampson, 1981). La gran mayoría de terapias cognitivas supone plantear este tipo de desafío para un paciente.

Para conseguir todo ello, las psicoterapias cognitivas se mueven en perspectivas que consideran a la terapia como una psicotecnología (Woolfolk, 1992). Toda la tecnología terapéutica de la que disponemos nos facilita superar aquello que nos aqueja. Hacemos terapia sin reflexionar sobre lo que esto implica, suponiendo que no es más que la aplicación neutral, ajena a valores, de todo un armamento tecnológico (Messer \& Woolfolk, 1998).

Sin embargo, y siguiendo a James y Prilleltensy (2002, pág. 1144) la psicoterapia no deja de ofrecer normas morales sobre lo que debe ser una buena persona o una buena familia. En este sentido, el paradigma cognitivo (cognitivo-conductual para los autores) asume una perspectiva pedagógica. Las ciencias sociales, sobre todo tras la II Guerra Mundial, nos ofrecen la educación necesaria para resolver los problemas sociales (Prilleltensky, 1990). Desarrollando esta idea, una buena 
persona, lee y rellena cuestionarios, siendo capaz de estimar sus conductas. Además, es racional, asertiva, tiene una elevada auto-estima y puede ser capaz de ofrecer explicaciones causales de su conducta. Si el paciente no es capaz de hacerlo, está siendo irracional o carece de las mínimas habilidades sociales. Ante esta persona se levanta todo este sistema psicotecnológico (Woolfolk, 1992), extremadamente apropiado dentro de una sociedad de máxima tecnificación (y mercantilización) del conocimiento (Garzón, 2012).

En definitiva, partiendo del énfasis, ya señalado, en el pragmatismo y en el funcionalismo, que suponen buena parte de la base de las psicoterapias cognitivas, se necesita desarrollar técnicas y demostrar su eficacia (Messer y Warren, 1990). No nos extraña, pues, el interés de las psicoterapias cognitivas clásicas por aparecer en los listados de "tratamientos empíricamente validados" (p.ej., Hoffman, et al., 2012; Lyddon y Jones, 2001) y su seguimiento de esta tendencia en el campo psicológico, llegándose a considerar la necesidad de ofrecer tratamientos eficaces y efectivos un imperativo social (Dobson, 2012, pág.112). ${ }^{2}$

La evolución de las psicoterapias cognitivas clásicas hace visible esta cuestión señalada ya hace años. Se articula un bagaje terapéutico que nos guía e instruye sobre lo que hacer de manera sistemática y manualizada (Kuyken, Padesky y Dudley, 2009). La insistencia en la correcta competencia en las distintas habilidades terapéuticas no deja de reflejar una cuestión ética de despliegue adecuado de nuestro conocimiento y una obligación moral incardinada socialmente. Este bagaje terapéutico requiere la participación activa del paciente. Un ejemplo de ello lo tenemos en el empleo de tareas y actividades en psicoterapia cognitiva.

\section{Las tareas y las actividades en psicoterapias cognitivas}

Uno de los elementos principales de las psicoterapias cognitivas es la insistencia en el desarrollo y seguimiento de tareas extra-sesión y de actividades intrasesión, que suponen que el paciente acude a psicoterapia a aprender qué hacer. Para ello está el terapeuta. Son innumerables los textos cognitivos donde esto se plantea de una forma habitual y se ejemplifica con el desarrollo y el seguimiento de casos clínicos. Pero para seguir con la línea de este trabajo debemos centrarnos en el marco del cual se deriva.

Las tareas y actividades ejemplifican la ética Protestante que es un elemento constitutivo de la sociedad americana. Prilleltensky (1990) relaciona esta ética con las contribuciones clave de Sampson (1977) y de Bellah, Madsen, Sullivan, Swidler y Tipton (1985). Para Sampson (1977) el ideal americano es un sí mismo supremo, es decir, un individuo omnipotente que tiene el poder de afrontar las desgracias por él mismo. El trabajo duro y la determinación, a pesar de los obstáculos, nos van a conducir a la prosperidad (Bellah, et al., 1985). Siguiendo con nuestro análisis, las tareas y actividades supondrían un ejemplo de este trabajo duro y del locus de control interno tan idealizado por los psicólogos (Prillestenky, 1990) y los terapeutas.

Analizando la responsabilidad personal y la autonomía, Rychlak (2003, pág. 
73) señalaba que el individualismo, para Thomas Jefferson (1743-1826), estaba basado, en parte, en los derechos naturales de los seres humanos. El individuo, entonces, tiene la libertad para vivir de forma auto-suficiente dentro de una sociedad. Esto nos lleva a suponer la necesidad de ser personalmente responsables, lo cual supone, a su vez, tener autonomía para gobernarnos usando el razonamiento, la evaluación y la elección. Para un paciente que comparta el mismo marco sociocultural que su terapeuta, no le va a ser extraño poner en marcha sus habilidades de razonamiento, evaluación y elección, en sesión, más allá de la propia sesión de terapia. La búsqueda de la comprensión y del distanciamiento frente a nuestros propios pensamientos, emociones y conductas nos permite poner en práctica lo que el psicoterapeuta cognitivo nos está dando. $\mathrm{Y}$ esto, en gran medida, toma la forma de trabajo dentro y fuera de sesión.

Por tanto, y volviendo al individualismo, esta tradición Protestante y Calvinista nos ha llegado, además, en forma de trabajo duro. Nuestra habilidad y nuestra disposición a trabajar determinan nuestra fortuna (Throop, 2009). Este es el reto implícito cuando se plantean tareas y actividades a un paciente. Algo así como: "en tus manos está el resultado de esta psicoterapia”.

\section{El sujeto cognitivo}

En un trabajo anterior (Caro Gabalda, 2015) planteábamos las implicaciones de considerar al paciente en terapia cognitiva como un sujeto, como el sujeto de una serie de acciones apropiadas para su marco terapéutico. Así lo concretábamos en un sujeto introspectivo y consciente (el que accede a su marco cognitivo); en un sujeto que es un indigente cognitivo (el que entra en psicoterapia cognitiva para recibir ayuda); y finalmente, el científico entrenado (aquel que se busca en psicoterapia cognitiva clásica).

En este apartado final, nos centraremos en la figura del sujeto cognitivo desde el punto que evidencia la reflexión que hemos hecho desde esta perspectiva sociocultural. Basándonos, en parte, en Markus y Kitayama (2010, pág. 428), deseos de la clase media americana y contextos europeos occidentales, como son el dominio, el control, el logro, la elección, la auto-expresión, y la unicidad, se manifiestan en nuestro contexto terapéutico.

El sujeto cognitivo es un sujeto motivado y agente, que trabaja duro. Un sujeto que tiene a su disposición los elementos para ejercer el control de su mundo privado e individual. Y para ello cuenta con un aliado inestimable, su terapeuta, que le guía, lo instruye, lo alecciona, lo acompaña, y lo comprende a la hora de recorrer su camino. Las metas terapéuticas son obvias.

La meta del modelo cognitivo consiste en acceder y trabajar con la vida interior gracias a la ayuda de un experto que pone en marcha intervenciones de tipo didáctico, desde una perspectiva científica y prescriptiva, y no biográfica o exploratoria (Pilgrim, 1997). Siendo esto muy representativo del modelo cognitivo debemos señalar que lo es más del modelo clásico que del modelo más constructivista. 
Los modelos constructivistas suavizan, por así decirlo, la figura didáctica del terapeuta asumiendo un papel más exploratorio y de persuasor o perturbador orientado estratégicamente (Guidano, 1991b). No obstante, el trabajo con la vida interior es inevitable, también para el contructivismo en terapia. Para lo que estamos planteando, da igual trabajar con emociones que con cogniciones, da igual inclinarnos por lo racional que por lo emocional, focalizarnos en el pensamiento o en la narración. En cualquier caso, se necesita un individuo que bucee en su interior, en su presente, en su pasado y en su futuro y lo aborde de forma diferente para lograr un cambio. Esta es la meta a la que se enfrenta el sujeto cognitivo.

Si tomamos la figura del héroe tan querida en el marco norteamericano (Bellah et al., 1985; Spence, 1990), podríamos asumir que este tipo de sujeto representa a un héroe que es capaz de seguir este camino individual hacia el logro y el control a pesar de los obstáculos. Se cae, pero se levanta, y sus esfuerzos se ven recompensados al final.

Sin embargo, cualquiera pensaría que esta cuestión del posible sujeto cognitivo no es generalizable a todo tipo de pacientes. Por ejemplo, ¿qué tipo de sujeto puede ser un paciente con diagnóstico de esquizofrenia? Bien, tomemos dos cuestiones en consideración. Por una parte algunas teorías actuales sobre la esquizofrenia y, por otra, el tipo de tratamiento que se da a estos pacientes en las terapias cognitivas clásicas.

¿Qué nos dicen las teorías metacognitivas actuales? Que el individuo piensa "mal", al ser incapaz de pensar sobre lo que piensa, y tomar perspectiva frente a ello. Los problemas metacognitivos de un paciente con esquizofrenia supondrían grandes dificultades para pensar sobre el pensamiento. Eso incluiría: hacer representaciones de sus propios estados mentales o de los otros; formar, cuestionar y revisar ideas sobre lo que se cree, siente, sueña, teme, etc. en contextos que cambian rápidamente (Lysaker, 2010). ¿Cómo se pone remedio? Enseñándole y entrenándole en habilidades metacognitivas para darse cuenta de uno mismo, de los otros, lograr descentrarse y darse cuenta de él mismo como alguien que confronta y afronta los desafíos psicológicos (Semerari, et al., 2003). El procedimiento terapéutico es de corte integrador y supone un proceso complejo que busca que el paciente se dé cuenta, y discuta con su terapeuta elementos importantes de sus acontecimientos vitales. El paciente debe darse cuenta y reflexionar (Lysaker, 2010). Algunos de estos elementos terapéuticos suponen (en Lysaker et al., 2014) el establecimiento de una excelente relación terapéutica que dé pre-eminencia a la agenda del paciente; la búsqueda de narraciones para que el paciente sea capaz de saber lo que pasa, lo comprenda y, en consecuencia, pueda nombrar el problema psicológico que tiene. Es un elemento clave la figura del terapeuta ya que se busca que el paciente reflexione sobre los procesos interpersonales que se dan en la sesión en ese diálogo continuo que se establece entre paciente y terapeuta. A medida que se progresa en la terapia, el paciente va a ser capaz de reflexionar sobre el progreso y se va a buscar y estimular la auto-reflexividad, el dominio y darse cuenta del otro. De nuevo, un 
individuo que debe mirar en su interior y mejorar sus déficits en la comprensión y conocimiento de la realidad.

Por otro lado, el bagaje terapéutico de las terapias cognitivas clásicas (véase Davidson, Lambert y McGlasham, 2004) reproduce el patrón "de acción” que estamos aquí desarrollando. En este contexto es muy importante el establecimiento de una agenda y el descubrimiento guiado como marco para trabajar y explorar creencias de los pacientes y comprenderlas. El trabajo con estos pacientes modifica y adapta la teoría clásica de Beck (1976) que postula técnicas orientadas al problema y encaminadas a cambiar errores o sesgos en las cogniciones que surgen al evaluar situaciones y modificar supuestos y creencias sobre uno mismo, el mundo y el futuro (Kingdom y Turkington, 2002). El modelo clásico con estos pacientes requiere de una buena colaboración y relación paciente-terapeuta y al mismo tiempo educar al paciente (Steel y Smith, 2013).

Se emplean técnicas para sustituir creencias desadaptativas por otras mejores y más funcionales tales como estrategias de desafío verbal para introducir la duda frente a las creencias; experimentos conductuales y exámenes planificados de la realidad que doten de prueba y comprobación a las creencias delirantes que se convierten en hipótesis a comprobar, por ejemplo. Incluso se emplea el ABC para hacer ver a los paciente qué influye (B) en sus emociones y comportamientos (C), experimentados en distintas situaciones (A) y para el análisis de sus alucinaciones (Chadwick, Birchwood y Trower, 1996; Perris, 2004). Así, estos pacientes también hacen tareas o se emplean registros y diarios sencillos, por ejemplo, de las voces experimentadas por un paciente a lo largo de la semana (Turkington, 2009).

Sigue estando a la base la figura del sujeto que intenta avanzar a pesar de las dificultades del camino, aunque éste no es tan optimista como el que se deduce del trabajo con pacientes en otras categorías diagnósticas. Es un sujeto, héroe por seguir con la metáfora, más atormentado, cuyo camino va a ser más complejo, doloroso, y difícil de recorrer y por tanto, más heroico. No obstante, si juzgamos por el tratamiento cognitivo estándar y meta-cognitivo con estos pacientes, el terapeuta no deja de asumir que este camino se puede y se debe recorrer. Se reproduce y ejemplifica, de nuevo y de esta manera, el ideario de la sociedad occidental que hemos desarrollado a lo largo de este trabajo.

\section{Conclusión}

Las psicoterapias cognitivas reproducen características socio-culturales de su particular momento histórico. Lo heredan y lo revalidan. La interacción entre sistema de psicoterapia y marco socio-cultural es directa, lo que aconseja escribir la historia de cada particular modelo psicoterapéutico en función de qué elementos socio-culturales se benefician y asumen, y no sólo de qué otras teorías han tomado sus principales premisas y conceptos. Los terapeutas somos ejecutores del poder y del control social, y no "aplicamos" técnicas solamente, sino que "instauramos" valores. Como terapeutas cognitivos podemos entender que lo que hacemos 
contribuye a una sociedad donde se privilegia el control por parte del individuo (foco principal) de la terapia, de sus problemas, fomentando la búsqueda en nuestro interior y el desarrollo de ciertas habilidades "cognitivas".

Debemos estar especialmente atentos a la sociedad en la que vivimos y a cambios que en ella se están produciendo. La psicoterapia cognitiva como un producto cultural se va ajustando a todos estos cambios. Esta cuestión, por ejemplo, se puede ver ejemplificada en la tendencia hacia el mindfulness y otros desarrollos en la denominada $3^{\text {a }}$ ola (Hayes, 2004). Estas nuevas tendencias y terapias suponen un foco distinto y la visión de un nuevo sujeto diferente del de las principales terapias cognitivas que hemos expuesto en éste y en otros trabajos (Caro Gabalda, 2015).

Estas reflexiones, como ya se ha señalado, son escasas en nuestro campo. Sin embargo, lo que se está haciendo ya desde hace algunos años es comenzar a alegar, con el paso del tiempo, que no podemos repetir el tratamiento cognitivo estándar con pacientes de otras culturas y condiciones (Hodges y Oei, 2007; Rathod y Kingdom, 2009). Cada vez son mayores los ejemplos de esta adaptación y de esta apertura a practicar la psicoterapia cognitiva al margen del tipo "clásico" con el que podemos identificarlas, y que algunos autores han descrito como un paciente masculino, blanco y heterosexual (por ejemplo, véase Graham, Sorenson, y HayesSkelton, 2013; Pomerantz, 2011).

Pero estos desarrollos y adaptaciones no han surgido de la nada. Surgen o se deben enmarcar en un diferente contexto socio-cultural que somete a las psicoterapias cognitivas a cambios para acceder a un mayor grupo de individuos de la manera más respetuosa y eficaz. Entender, sugerimos, por qué estamos cambiando el foco, y de dónde viene nuestro foco principal, nos puede enriquecer como terapeutas.

\section{Notas}

1 El enfoque de Guidano es claramente post-racionalista, pero se apela a que el paciente logre entender y comprender qué le pasa, el cómo y el por qué (Guidano, 1991a).

2 Este interés por lo empíricamente validado no caracteriza a las psicoterapias cognitivas de corte constructivista. Su interés está en la demostración clínica de su uso y en la fundamentación de sus conceptos principales. La idea del Método, imperante en la tradición cognitiva principal, les es ajena por razones epistemológicas y ontológicas.

\section{Referencias bibliográficas}

Beck, A.T. (1976). Cognitive therapy and the emotional disorders. Nueva York: International Universities Press. Beck, A.T., Rush, A.J., Shaw, B.F., \& Emery, G. (1979). Cognitive therapy of depression. Chichester: Wiley.

Beck, A.T. \& Weishaar, M. (1989). Cognitive therapy. En A.T. Freeman, K.M. Simon, L.E. Beutler, y H. Arkowitz (eds.), Comprehensive handbook of cognitive therapy (pp. 21-36). Nueva York: Plenum Press.

Bellah, R.N., Madsen, R., Sullivan, W.M., Swidler, A. \& Tipton, S.M. (1985). Habits of the heart. Berkeley: University of California Press.

Bem, S. \& Keijzer, F. (1996). Recent changes in the concept of cognition. Theory \& Psychology, 6, 449-469.

Berger, P.L. (1965). Towards a sociological understanding of psychoanalysis. Social Research, 32, 26-41.

Berger, P.L. \& Luckmann, T. (1966). The social construction of reality. Londres, Reino Unido: Penguin. 
Brewin, C.R. (1989). Cognitive change processes in psychotherapy. Psychological Bulletin, 96, 379-394.

Buss, A.R. (1975). The emerging field of the sociology of psychological knowledge. American Psychologist, 30 , 988-1002.

Caro Gabalda, I. (1995). Pasado, presente y futuro de las terapias cognitivas. Boletín de Psicología, 46, 115-160.

Caro Gabalda, I. (2015). The subject in cognitive psychotherapy. Anales de Psicología, 31, 379-389. http:// dx.doi.org/10.6018/analesps.31.2.200201

Chadwick, P., Birchwood, M.J. \& Trower, P. (1996). Cognitive therapy for delusions, voices and paranoia. Oxford: Wiley.

Clark, D.A., Beck, A.T., \& Alford, B.A. (1999). Cognitive theory and therapy of depression. Nueva York: Wiley Press.

Cushman, P. (1992). Psychotherapy to 1992: A historically situated interpretation. En D.K. Freedheim (Ed.), History of psychotherapy. A century of change (pp. 21-64). Washington: APA.

Cushman, P. (1993). Psychotherapy as moral discourse. Journal of Theoretical and Philosophical Psychology, 13, 103-113.

Cushman, P. (2002). How psychology erodes personhood. Journal of Theoretical and Philosophical Psychology, 22, 103-113.

Cushman, P. \& Gilford, P. (2000). Will managed care change our way of being? American Psychologist, 55, $985-$ 996. doi: 10.1037//0003-066X.55.9.985

Davidson, L., Lambert, S \& McGlasham, T.H. (2004). Tratamientos psicoterapéuticos y cognitivo-conductuales para la esquizofrenia: desarrollo de una forma de psicoterapia específica del trastorno para personas con psicosis. En C. Perris y P.D. McGorry (Eds.), Psicoterapia cognitiva para los trastornos psicóticos y de personalidad (pp. 19-42). Bilbao, España: DDB (Original inglés de 1998).

Dobson, K.S. (2012). Cognitive therapy. Washington: APA.

Dobson, K. S. \& Dobson, K. (2009). Evidence-based practice of cognitive-behavioral therapy. Nueva York: Guilford Press.

Dow, J.W. (1986). Universal aspects of symbolic healing: A theoretical synthesis. American Anthropologist, 88, 56-69.

Dowd, E.T. (2002). History and recent developments in cognitive psychotherapy. En R.L. Leahy y E.T. Dowd (Eds.), Clinical advances in cognitive psychotherapy (pp.15-28). Nueva York: Springer.

Dryden, W. \& Ellis, A.(1988). Rational-emotive therapy. En K.S. Dobson (ed.), Handbook of cognitivebehavioural therapies (pp. 214-272). Londres: Hutchinson.

Eberle, T.S. (1993). Social psychology and the sociology of knowledge. Revista de Psicología Social, 8, 5-13.

Ehrenwald, J. (1969). Psychotherapy: Myth and method. Nueva York: Grune \& Stratton.

Ellis, A. (1962). Reason and emotion in psychotherapy. Nueva York: Stuart.

Ellis, A. (1973). Rational-emotive therapy. En R. Corsini (Ed.), Current psychotherapies (pp. 167-206). Itaca: F.E. Peacock.

Erwin, E. (1997). Philosophy and psychotherapy. Londres, Reino Unido: Sage.

Frank, J.D. (1961) Persuasion and healing. Baltimore: John Hopkins Press.

Garzón, A. (2012). Tecnificación del conocimiento y creencias sociales. Boletín de Psicología, 106, 29-44.

Gonçalves, O. (1994). Cognitive narrative psychotherapy: The hermeneutic construction of alternative meanings. Journal of Cognitive Psychotherapy, 8, 105-126.

Gónzalez Pardo, H. \& Pérez Álvarez, M. (2007). La invención de trastornos mentales. Madrid: Alianza Editorial.

Graham, J.R., Sorenson, S., \& Hayes-Skelton, S.A. (2013). Enhancing the cultural sensitivity of cognitive behavioral interventions for anxiety in diverse populations. Behavior Therapy, 36, 101-108.

Guidano, V. (1991a). The self in process. Nueva York: Guilford Press (trad. cast. en Ed. Paidós: El sí mismo en proceso, 1994).

Guidano, V.(1991b). Affective change events in a cognitive therapy system approach. En Safran, J.D. y Greenberg, L.S. (comps.), Emotion and the process of therapeutic change (pp. 50-79). Nueva York: Guilford Press.

Guidano, V. (1997). El estado de la cuestión en la terapia cognitiva postracionalista. En I. Caro (ed.), Manual de psicoterapias cognitivas (pp.371-380). Barcelona, España: Paidós.

Guidano, V.\& Liotti, G. (1988). A constructivist foundation for cognitive therapy. En M.J. Mahoney \& A. Freeman (Eds.), Cognition and psychotherapy (pp. 101-142). Nueva York: Springer.

Hansen, J.T. (2014). Philosophical Issues in counseling and psychotherapy. Encounters with four questions about knowing, effectiveness, and truth. Lanham: Rowan \& Littlefield.

Hayes, S.C. (2004). Acceptance and commitment therapy, relational frame theory, and the third wave of behavioral and cognitive therapies. Behavior Therapy, 35, 639-665. 
Hofmann, S.G., Asnaani, A., Vonk, I.J.J., Sawyer, A.T., \& Fang, A. (2012). The efficacy of cognitive behavioral therapy: A review of meta-analyses. Cognitive Therapy and Research, 36, 427-440. doi: 10.1007/s10608012-9476-1

Hofstede, G. (1980). Culture's consequences: International differences in work-related values. Beverly Hills: Sage Publications.

Hodges, J. \& Oei, T.P. (2007). Would Confucius benefit from psychotherapy? The compatibility of cognitive behaviour therapy and Chinese values. Behaviour Research \& Therapy, 45, 901-914. doi: 10.1016/ j.brat.2006.08.015

Hollon, S. D. \& DiGiuseppe, R. (2010). Cognitive theories of psychotherapy. En J.C. Norcross, G.R. Vandenbos, \& D. K. Freedheim (Eds.), History of psychotherapy. Continuity and change (pp. 201-241). Washington: APA.

Ibáñez, E. (1990). Presupuestos básicos y clasificación de las terapias cognitivas: Un punto de vista para la psiquiatría. Trabajo presentado en el Congreso Nacional de la Sociedad Española de Psiquiatría.

Ibáñez,E.(1993). Bosquejo para el análisis de las relaciones entre personalidad y psicoterapia. En I. Caro (ed.), Psicoterapia e investigación de procesos (pp. 351-369). Valencia, España: Promolibro. Colección de Psicología Teorética.

Ingram, R.E. \& Kendall, P.C. (1986). Cognitive clinical psychology: Implications of an information processing perspective. En R.E. Ingram (Ed.), Information processing approaches to clinical psychology (pp. 3-21). Orlando: Academic Press.

James, S. \& Foerster, G. (2006). Reconciling rules with context. An ethical framework for cultural psychotherapy. Theory \& Psychology, 16, 803-823. doi: 10.1177/0959354306070532

James, S. \& Prilleltensky, I. (2002). Cultural diversity and mental health. Towards integrative practice. Clinical Psychology Review, 22, 1133-1154.

Kazarian, S.D. \& Evans, D.R. (1998). Cultural clinical psychology. En S.S. Kazarian \& D.R. Evans (Eds.), Cultural clinical psychology: Theory, research, and practice (pp. 3-38). Nueva York: Oxford University Press.

Kingdom, D. \& Turkington, D. (2002). Introduction. En D. Kingdom \& D. Turkington(Eds.), The case study guide to cognitive behavior therapy of psychosis (pp. 1-10). Chichester: Wiley.

Kuyken, W., Padesky, C. \& Dudley, R. (2009). Collaborative case conceptualization. Nueva York. Guilford.

Lam, D.C.K. (2008). Cognitive behavioural therapy. A practical guide to helping people to gain control. Hove: Routdlege.

Landrine, H. (1992). Clinical implications of cultural differences: The referential versus the indexical self. Clinical Psychology Review, 12, 401-415.

Lyddon, W.J. \& Jones, J.V (2001) (Eds.). Empirically supported cognitive therapies. Nueva York: Springer.

Lysaker, P.H. (2010). Metacognition in schizophrenia spectrum disorders: methods of assessing metacognition within narrative and links with neurocognition. En G. Dimaggio \& P.H. Lysaker (Eds.), Metacognitive and severe adult mental disorder (pp. 65-82). Londres, Reino Unido: Routdlege.

Lysaker, P.H., Buck, K.F., Leonhardt, B.L. Buck, B, Hamm, J., Hasson-Ohayon, I., Vohs, J.L., \& Dimaggio, G. (2014). Metacognitively focused psychotherapy for people with schizophrenia: Eight core elements that define practice. En P.H. Lysaker, G. Dimaggio \& M. Brüne (Eds.), Social cognition and metacognition in schizophrenia. Psychopathology and treatment approaches (pp. 195-213). Amsterdam, Países Bajos: Elsevier.

Mahoney, M.J. (1985). Psychotherapy and human change processes. En M.J. Mahoney \& A. Freeman (Eds.), Cognition and psychotherapy (pp. 3-48). Nueva York: Springer.

Mahoney, M.J. (1988). Rationalism and constructivism in clinical judgment. En D.C. Turk \& P. Salovey (Eds.), Reasoning, inference, and judgment in clinical psychology (pp. 155-181). Nueva York: Free Press.

Mahoney, M.J. (1991). Human change processes. Nueva York: Basic Books.

Mahoney, M.J. (2005). Constructive psychotherapy. A practical guide. Nueva York: Guilford Press.

Markus, H.R \& Kitayama, S. (1991). Culture and the self: Implications for cognition, emotion, and motivation. Psychological Review, 98, 224-253.

Markus, H.R \& Kitayama, S. (2010). Cultures and selves: A cycle of mutual constitution. Perspectives on Psychological Science, 5, 420-430. doi: 10.1177/1745691610375557

Messer, S.B. \& Warren, S. (1990). Personality change and psychotherapy. En L. Pervin (Ed.), Handbook of personality. Theory and research (pp. 371-398). Nueva York: Guilford Press.

Messer, S.B. \& Woolfolk, R.L. (1998). Philosophical issues in psychotherapy. Clinical Psychology: Science \& Practice, 5, 251-263. 
Neimeyer, R.A. (1993). An appraisal of constructivist psychotherapies. Journal of Consulting and Clinical Psychology, 61, 221-234.

Norcross, J.C. (1986). Eclectic psychotherapy: An introduction and overview. En J.C. Norcross (Ed.), Handbook of eclectic psychotherapy (pp. 3-24). Nueva York: Brunner/Mazel.

Norcross, J.C. (2005). A primer on psychotherapy integration. En J. C. Norcross \& M. R. Goldfried (Eds.) Handbook of psychotherapy integration (2 ed.) (pp. 3-23). Nueva York: Oxford University Press.

Ogihara, Y. \& Uchida, Y. (2014). Does individualism bring happiness? Negative effects of individualism on interpersonal relationships and happiness. Frontiers in Psychology, 5, 1-8. doi: 10.3389/fpsyg.2014.00135

Owen, I.R. (1993). Towards a sociology of psychotherapy. Counselling Psychology Review, 8, 6-9.

Perris, H. (2004). Estrategias y técnicas terapéuticas menos comunes en la psicoterapia cognitiva de pacientes con trastornos graves. En C. Perris \& P.D. McGorry (Eds.), Psicoterapia cognitiva para los trastornos psicóticos y de personalidad (pp. 379-394). Bilbao, España: DDB (Original inglés de 1998).

Pilgrim, D. (1997). Psychotherapy and society. Londres, Reino Unido: Sage.

Pinillos, J.L. (1997). El corazón del laberinto. Madrid, España: Espasa.

Polkinghorne, D.E.(1992). Postmodern epistemology of practice. En S. Kvale(Ed.), Psychology and postmodernism (pp. 146-165). Londres, Reino Unido: Sage.

Pomerantz, A.M. (2011). Clinical psychology. Science, practice and culture (2a Ed.). Los Angeles: Sage.

Prilleltensky, I. (1989). Psychology and the status-quo. American Psychologist, 44, 795-802.

Prilleltensky, I. (1990). On the social and political implications of cognitive psychology. The Journal of Mind and Behavior, 11, 127-136.

Prilleltensky, I. (1997). Values, assumptions, and practices. Assessing the moral implications of psychological discourse and action. American Psychologist, 52, 517-535.

Rathod, S. \& Kingdon, D. (2009). Cognitive behavior therapy across cultures. Psychiatry, 8, 370-371. doi.org/ 10.1016/j.mppsy.2009.06.011

Robinson, D.N. (1981). An intellectual history of psychology. Nueva York: McMillan.

Robinson, D.N. (1997). Therapy as theory and as civics. Theory \& Psychology, 7, 675-681.

Rohner, R.P. (1984). Toward a conception of culture for cross-cultural psychology. Journal of Cross-cultural Psychology, 15, 111-138.

Rychlak, J. F. (2003). The human image in postmodern America. Washington: APA.

Sampson, E.E. (1977). Psychology and the american ideal. Journal of Personality and Social Psychology, 35, 767782.

Sampson, E.E. (1981). Cognitive psychology as ideology. American Psychologist, 36, 730-743.

Schirrmacher, F. (2014). Ego. Las trampas del juego capitalista. Barcelona, España: Ariel. (Original alemán de 2013).

Semerari, A., Carcione, A., Dimaggio, G., Falcone, M., Nicolò, G., Procacci, M., et al. (2003). How to evaluate metacognitive functioning in psychotherapy? The metacognition assessment scale and its applications. Clinical Psychology and Psychotherapy, 10, 238-261. doi: 10.1002/cpp.362

Seoane, J. (2005). Hacia una biografía del self. Boletín de Psicología, 85, 41-87.

Smith, R. (1997). The Norton history of the human sciences. Nueva York: Norton.

Spence, D.P. (1990). Theories of the mind: Science or literature? Poetics Today, 11, 329-347.

Steel, C. \& Smith, B. (2013). CBT for psychosis: An introduction. En C. Steel (Ed.), CBT for schizophrenia: Evidence-based interventions and future directions (pp. 4-12). Nueva York: Wiley.

Throop, E. (2009). Psychotherapy, American culture and social policy. Immoral individualism. Nueva York: Palgrave.

Toukmanian, S.G. \& Brouwers, M.C. (1998). Cultural aspects of self-disclosure and psychotherapy. En S.S. Kazarian \& D.R. Evans (Eds.), Cultural clinical psychology. Theory, research and practice (pp. 106-124). Nueva York. Oxford University Press.

Triandis, H.C. (1995). Individualism and collectivism. Boulder: Westview.

Turkington, D. (2009). Voices. En D. Turkington, et al. (Eds.), Back to life, back to normality. Cognitive therapy, recovery and psychosis (pp. 61-78). Cambridge: Cambridge University Press.

Wampold, B.E. (2001). Contextualizing psychotherapy as a healing practice: Culture, history, and methods. Applied \& Preventive Psychology, 10, 69-86. doi: 10.1017/S0962-1849(02)01001-6

Watts, A. (1961). Psychotherapy east and west. Nueva York: Pantheon Books.

Woolfolk, R.L. (1992). Hermeneutics, social constructionism, and other items of intellectual fashion: Intimations for clinical science. Behavior Therapy, 23, 213-223.

Young, J.E., Klosko, J.S. \& Weishaar, M.E. (2003). Schema therapy. A practitioner's guide. Nueva York: Guilford Press. (Edición en castellano, DDB). 